\title{
Deep morphologies of type I planetary nebulae ${ }^{\star}$
}

\author{
C.T. Hua
}

Laboratoire d'Astronomie Spatiale du CNRS, Allée Peiresc, Traverse du Siphon, 13012 Marseille, France

Received February 23, 1996; accepted January 13, 1997

\begin{abstract}
Deep narrow band interference filters observations are presented for 7 type I galactic planetary nebulae $(\mathrm{PNe})$. Except for the nearly circular planetary nebula A 39, the observations show overall dimensions appreciably larger than those quoted so far. The observed PN (NGC 6302 excepted) could have linear diameters larger than 1 pc. Our new observations imply ionized masses often larger than $1 M_{\odot}$, suggesting that much of the mass ejected by the PN precursor is contained in faint outer extensions. This might contribute to the solution of the "mass deficit" in PNe. We have also provided calibrated fluxes for some specific emission lines. In spite of their apparent morphological differences, our observations suggest that these type I PNe possess the same intrinsic structure as that displayed by NGC 650-1, the Ring Nebula, and even Sh 1-89 with its elongated jet-like feature, but viewed under different angles.
\end{abstract}

Key words: planetary nebulae

\section{Introduction}

Since PN morphology is presumably connected with the evolution of the ensemble PN - central star, available observations obviously show a large variety of shapes, which could reflect different stages of this evolution. Progress in theories (Frank et al. 1993; Bryce et al. 1994) requires a large, homogeneous set of monochromatic images and isophotes (Kaler 1985) which narrow bandpass CCD imaging of PN can supply (Balick 1987).

Here we present new sensitive observations using selective filters for seven galactic type I PN (Peimbert 1978; Peimbert \& Serano 1980) which are characterized by high helium and nitrogen contents and which are believed to originate from massive precursor stars (Peimbert

\footnotetext{
* The observations were carried out at the Observatoire de Haute Provence, St Michel l'Observatoire - France. (*) NGC 6302 was observed at Mt John University Observatory - Lake Tekapo - operated by the University of Canterbury, Christchurch, New-Zealand.
}

\& Peimbert 1983). Our goal in this study was to search for the so-called secondary structures, if any, around the bright central nebula, and which could be relics of primary ejections by the evolving ionizing star in the AGB phase. The overall diameter measured on the $\mathrm{H}_{\alpha}$ monochromatic images should allow new estimates of the ionized mass of the planetary nebula. As a matter of fact, existing inventories of this parameter in $\mathrm{PN}$ reveal a shortfall as compared with theory.

\section{The observational material}

Six northern planetary nebulae were observed with a Tektronic CCD installed at the $\mathrm{f} / 6$ focus of the $120-\mathrm{cm}$ telescope of the Haute Provence Observatory: it offers a total field of view of $390 \times 390$ square $\operatorname{arcsec}(27 \mu \mathrm{m}$ pixel-size or 0.77 arcsec pixel $\left.{ }^{-1}\right)$. The southern object, NGC 6302, was observed with the McLellan 1-m telescope (f/7.8) at Mt John, New Zealand, using a Thomson TH7882 CDAchip $\left(384 \times 57623 \mu \mathrm{m}\right.$ square pixels, 0.61 arcsec pixel $\left.{ }^{-1}\right)$ covering a total field of view of $240 \times 360$ square arcsec.

The interference filters used in our study have bandwidths $\leq 10 \AA$, with typical transmission of about $60 \%$. In some cases, however, $44 \AA$-wide $\mathrm{H}_{\alpha}$, [OIII] $5007 \AA$ and $39 \AA$-wide [SII] $6737 \AA$ filters were used. The filters were mounted in front of the detectors in the direct imaging mode. The image quality was about $1^{\prime \prime} .5-2^{\prime \prime}$ during the observations.

\section{Results}

Table 2 displays line intensity measurements taken from Acker et al. 1993 (hereafter Acker93) and Cahn et al. 1992 (CKS92) as well as other published parameters collected from the literature for seven $\mathrm{PN}$ in study. Peimbert \& Peimbert 1983 defined as type I PN those having $N(\mathrm{He}) / N(\mathrm{H}) \geq 0.125$ or $\mathrm{N} / \mathrm{O} \geq 0.5$, and which generally display bipolar structures. Table 2 summarizes the measured parameters ( $\mathrm{PN}$ dimensions, ionized mass in solar mass, and absolute fluxes). 
Table 1. Characteristics of observed PN

\begin{tabular}{lrrrrrrrrrrr}
\hline Names & $\mathrm{H}_{\alpha}$ & {$[\mathrm{NII}]$} & {$[\mathrm{OIII}]$} & $\mathrm{He} / \mathrm{H}$ & {$[\mathrm{NII}] / \mathrm{H}_{\alpha}$} & $C_{\mathrm{H} \beta}$ & $\left.\mathrm{Size}^{\prime \prime}\right)$ & $12 \mu \mathrm{m}$ & $25 \mu \mathrm{m}$ & $60 \mu \mathrm{m}$ & $100 \mu \mathrm{m}$ \\
\hline A 39 & - & - & - & 0.110 & - & 0.0 & 174 & 0.50 & 0.25 & 0.90 & 1.46 \\
Sh 1-89 & 639.00 & 823.00 & 433.00 & 0.193 & 1.288 & 0.86 & 35 & 0.89 & 0.25 & 2.14 & 20.15 \\
Sh 2-71 & 1088.00 & 3888.00 & 1423.00 & 0.110 & 3.574 & 1.45 & 100 & 1.41 & 2.26 & 4.50 & 102.18 \\
N650-1 & 649.00 & 490.00 & 1186.00 & 0.108 & 0.755 & 0.20 & 67 & 0.28 & 2.79 & 6.80 & 9.29 \\
N6302 $^{*}$ & 704.00 & 1758.00 & 1354.00 & 0.182 & 2.497 & 1.26 & 44.5 & 32.08 & 335.90 & 849.70 & 537.40 \\
N6543 $_{(c)}$ & $323.30^{* *}$ & 20.00 & 788.70 & 0.111 & 0.062 & 0.12 & 19.5 & 7.52 & 113.48 & 133.40 & 62.68 \\
N6543 $_{(h)}$ & $323.40^{* *}$ & 304.80 & 1895.00 & - & 0.942 & & - & 7.52 & 113.48 & 133.40 & 62.68 \\
N6720 $^{2}$ & 299.00 & 297.00 & 1064.00 & 0.113 & 0.993 & 0.29 & 76 & 0.82 & 10.15 & 52.27 & 54.62 \\
\hline
\end{tabular}

The line intensity values (normalized to $F\left(\mathrm{H}_{\beta}\right)=100$ ) are from Acker 1993's tables when available. (**) Data from Manchado $\&$ Pottasch 1989; indices (c) and (h) being related to the centre and the halo nebula respectively. $C_{\mathrm{H} \beta}$ are optical extinction values at $\mathrm{H}_{\beta}$ from CKS92 (their $C_{\alpha}$ column) and IRAS data from the IRAS catalogue. NGC 6302 was observed at the MJUO McLellan 1-m telescope.

Table 2. Measured parameters of observed PN

\begin{tabular}{|c|c|c|c|c|c|c|c|}
\hline Names & $\mathrm{H}_{\alpha}\left({ }^{\prime \prime}\right)$ & $F\left(\mathrm{H}_{\alpha}\right)$ & $F([\mathrm{NII}])$ & $F([\mathrm{OIII}])$ & $d(\mathrm{pc})$ & $M_{i}(*)$ & $-\log F\left(\mathrm{H}_{\beta}\right)$ \\
\hline$\overline{A ~ 39}$ & 175 & 5.15 & - & 19.94 & 1163 & 0.017 & 11.75 \\
\hline Sh 1-89 & $\sim 400 \times 110$ & 8.81 & 8.62 & 2.76 & 1941 & 0.235 & \\
\hline Sh $\left.2-71_{(} c\right)$ & $250 \times 300$ & 26.37 & 37.2 & 11.0 & 997 & 0.047 & 11.63 \\
\hline N650-1 & 300 & 86.14 & 81.31 & & 739 & 0.022 & 10.87 \\
\hline N6302* & $>250$ & 210 & & & 525 & $*$ & 10.55 \\
\hline $\operatorname{N6543}_{(c)}$ & $30 \times 25$ & 902.60 & 104.35 & 1952.70 & 982 & & 9.63 \\
\hline $\mathbf{N 6 5 4 3}_{(h)}$ & 340 & 24.0 & 5.3 & 41.1 & 982 & 0.079 & 11.15 \\
\hline N6720 & 260 & 327 & 290 & - & 872 & 0.02 & 10.01 \\
\hline
\end{tabular}

Fluxes are given in $10^{-12}$ erg $\mathrm{cm}^{-2} \mathrm{~s}^{-1} \cdot\left(^{*}\right)$ To evaluate $\mathrm{PN}$ ionized mass in $M_{\odot}$, multiply these figures by the corresponding values of filling factor $\epsilon$ and electron density. Distances are in parsecs from CKS92. ${ }^{*}$ See text.

\subsection{A 39: $P N 047.0+42.4$}

The $\mathrm{H}_{\alpha}$ and [OIII]5007 $\AA$ images display a nearly circular and identical shape (Figs. 1). However, this circular symmetry seems to be distorted ${ }^{1}$ by the interaction with the interstellar medium. The diameter measured on the $\mathrm{H}_{\alpha}$ image $\left(\sim 175^{\prime \prime}\right)$ agrees with the value of $174^{\prime \prime}$ reported by Cahn \& Kaler (1971) (CK71). [NII]6583 emission was not detected. At the distance of 1200 pc (CKS92), the observed diameter corresponds to a linear value of $1.0 \mathrm{pc}$.

\subsection{Sh $1-89$ : $P N 089.8-00.6$}

This object was not detected with the filter [OII $] 3727$ (1800 s), and curiously HeII (600 s) as well, but we strongly suspect the effets of the bad weather conditions during these observations. The $\mathrm{H}_{\beta}$ images are not usable for investigating internal absorption. Nevertheless, all the other images (Figs. 2) display outstanding peripheral features, the nebular dimensions being definitely larger than $35^{\prime \prime}$ quoted. The observed $\left(\mathrm{H}_{\alpha}\right.$ and $\left.[\mathrm{NII}]\right)$ images show a rather complex structure. Even the bright core actually

\footnotetext{
1 We used a POLAR treatment to evidence the curvature of the borders.
}

spreads over $60^{\prime \prime}$. Bubbles and wisps extend over 6 arcminutes. Due to the faint jet-like emissions on both sides of the bright waist, we can hardly assign to Sh 1-89 any of the available models (bubble, butterfly, jet?) to explain such a peculiar morphology. The overall dimensions are $\sim 400^{\prime \prime}$ along the major axis. Sh $1-89$ would be one of rare planetary nebulae which have linear diameter larger than $1 \mathrm{pc}$, since its main bubble-like structure spreads over $230^{\prime \prime}\left(1^{\prime \prime}=9.4110^{-3} \mathrm{pc}\right)$.

\section{3. $S h 2-71$ : $P N 035.9-01.1$}

The $\mathrm{H}_{\alpha},[\mathrm{NII}] 6583 \AA,[\mathrm{OIII}] 5007 \AA$ (Figs. 3), $\mathrm{H}_{\beta}$, and $39 \AA$-wide [SII] $6737 \AA$ images show a rather elongated and bright central nebula $\left(130^{\prime \prime} \times 220^{\prime \prime}\right)$, whereas an outer faint emission spreads over a larger area $250^{\prime \prime} \times 300^{\prime \prime}$ than previously quoted (100", CK71). The extensions reported in this study are even much larger than that reported by Jewitt et al. 1986 who considered Sh $2-71$ as an excellent example of PN with halo even though these authors observed only the bright elongated core. The nitrogen emission is more prominent along the radial structures outwards. The $\mathrm{H}_{\alpha}$, [NII] $6583 \AA$ images display a bright elongated nebula $\left(\sim 130^{\prime \prime} \times 220^{\prime \prime}\right)$, with faint emission 


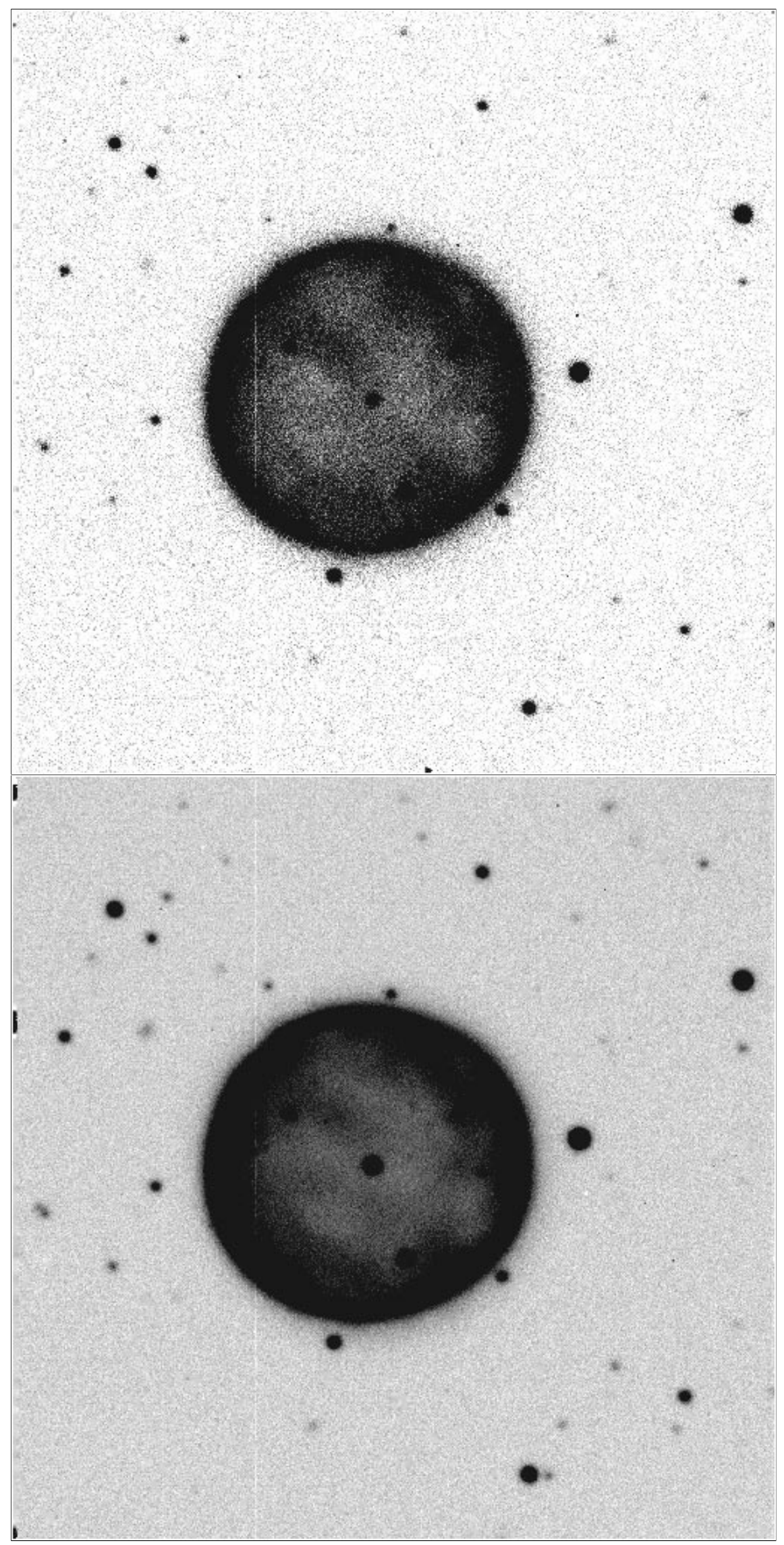

Fig. 1. a) Top: $\mathrm{H} \alpha$. The planetary nebula shows a nearly circular shape. At the distance of $1200 \mathrm{pc}$, A 39 has a linear diameter of $\sim 1$ pc. The overall field of view covers $386 \times 386$ arcseconds. North is towards the top and East at left for all figures. b) Bottom: The [OIII] emission is much stronger than that in $\mathrm{H}_{\alpha}$ 


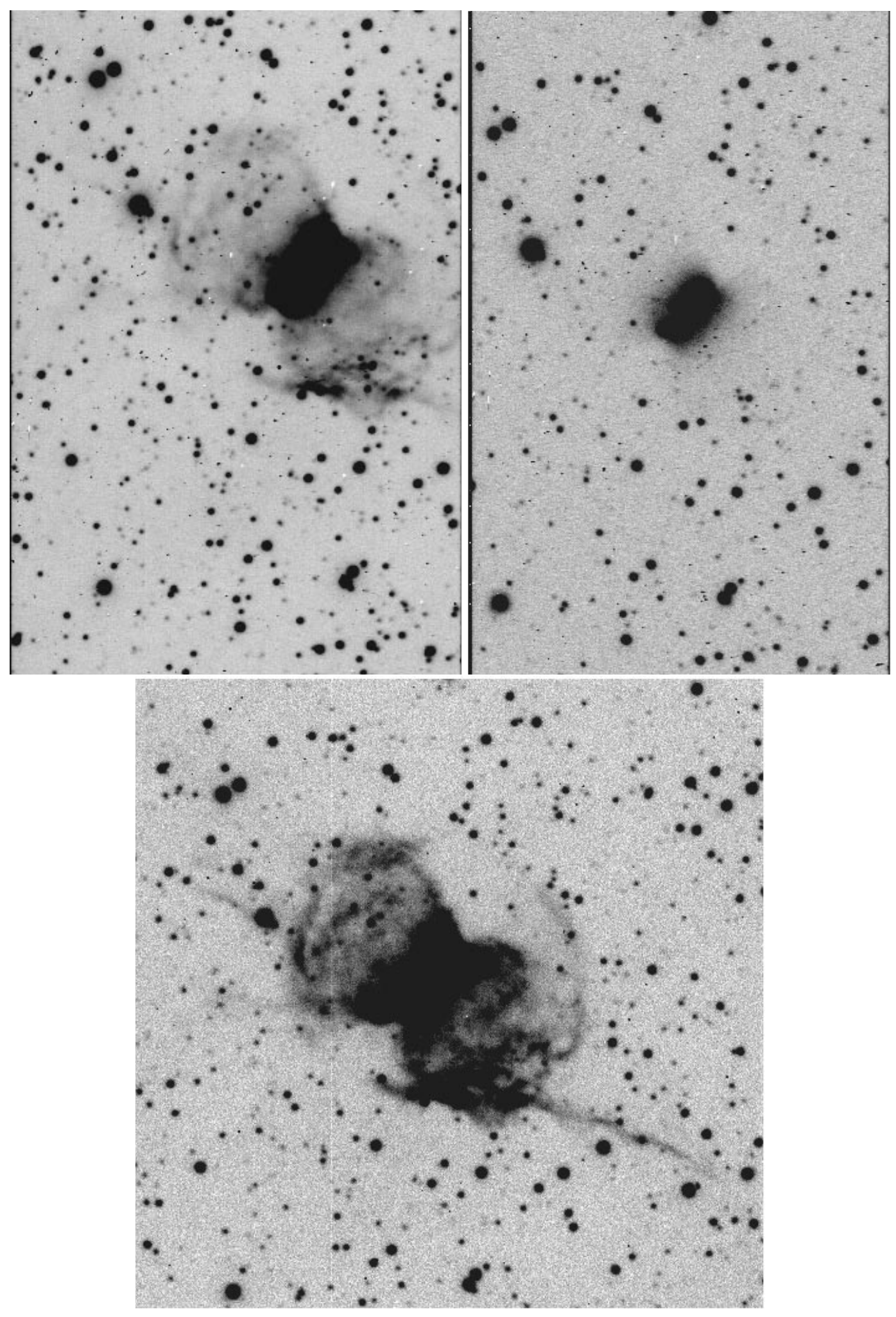

Fig. 2. a) Top left: The $\mathrm{H}_{\alpha}$ image was obtained with a RCA-CCD. Due to the limited dimensions of the detector $(320 \times 512$ i.e. $260^{\prime \prime} \times 418^{\prime \prime}$ ) the extended western filament is not shown here. A substantial contribution from the nitrogen emission line should be taken into account into the $48 \AA$ wide bandpass. b) Bottom: Obtained with a Tektronic $512 \times 512$ CCD: the bright centre spreads over $\sim 60^{\prime \prime}$. The total field of view covers $390 \times 390$ square arcseconds. Note the "jet-like" structure on both sides of the main bubbles marked by filamentary network. The major axis extends up to $\leq 400^{\prime \prime}$, whereas the main bubble-like nebula spreads over $230^{\prime \prime}(2.16 \mathrm{pc})$. c) Top right: Only the bright centre is seen here, surrounded by a rather diffuse emission 


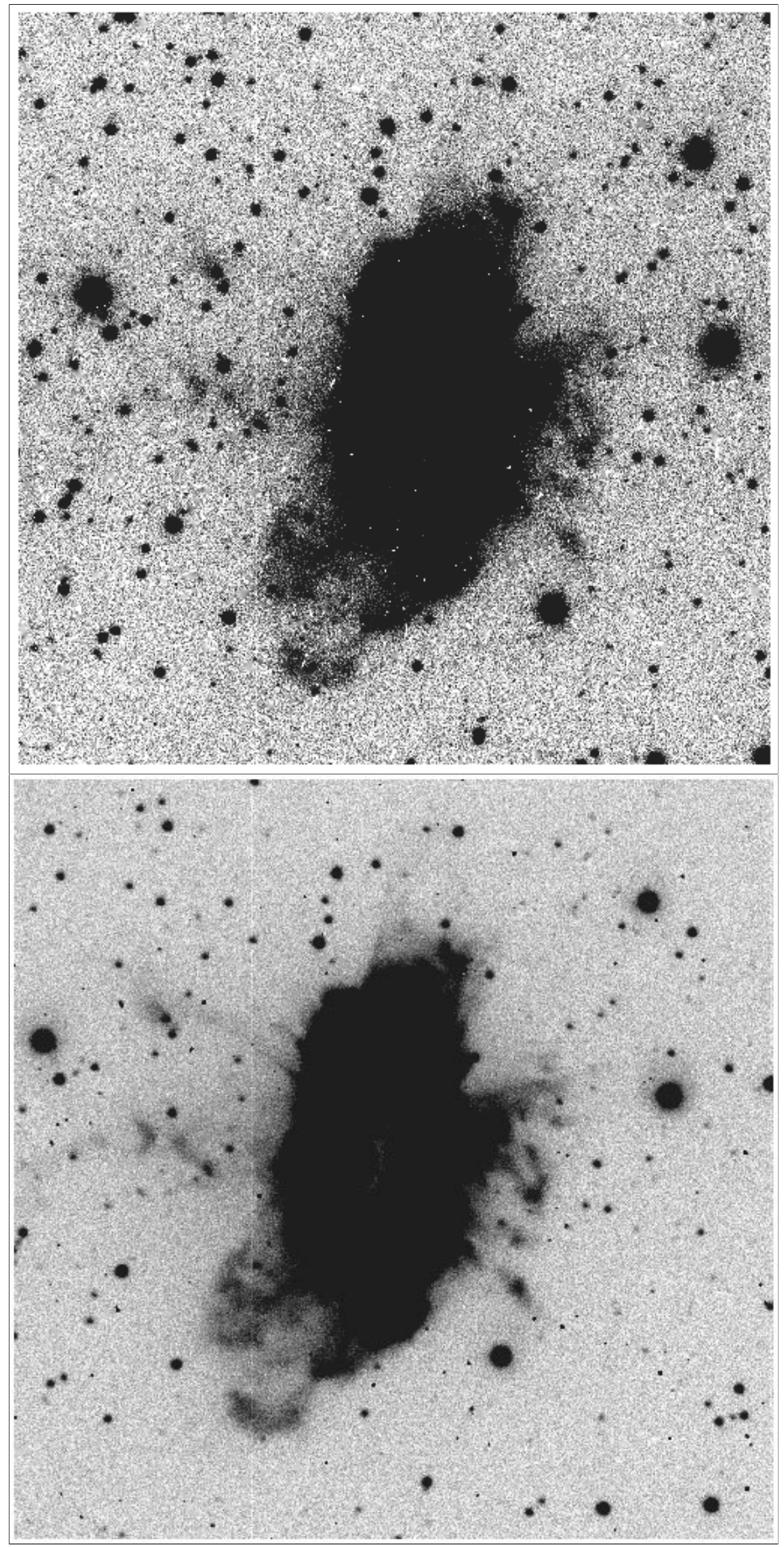

Fig. 3. a) Top: $\mathrm{H} \alpha$. The overall dimensions spread over $250^{\prime \prime}$ or $1.2 \mathrm{pc}$. The bright elongated core is surrounded by a faint envelope. b) Bottom: The outer [NII]6583 emission is more conspicuous than that seen in $\mathrm{H}_{\alpha}$ 


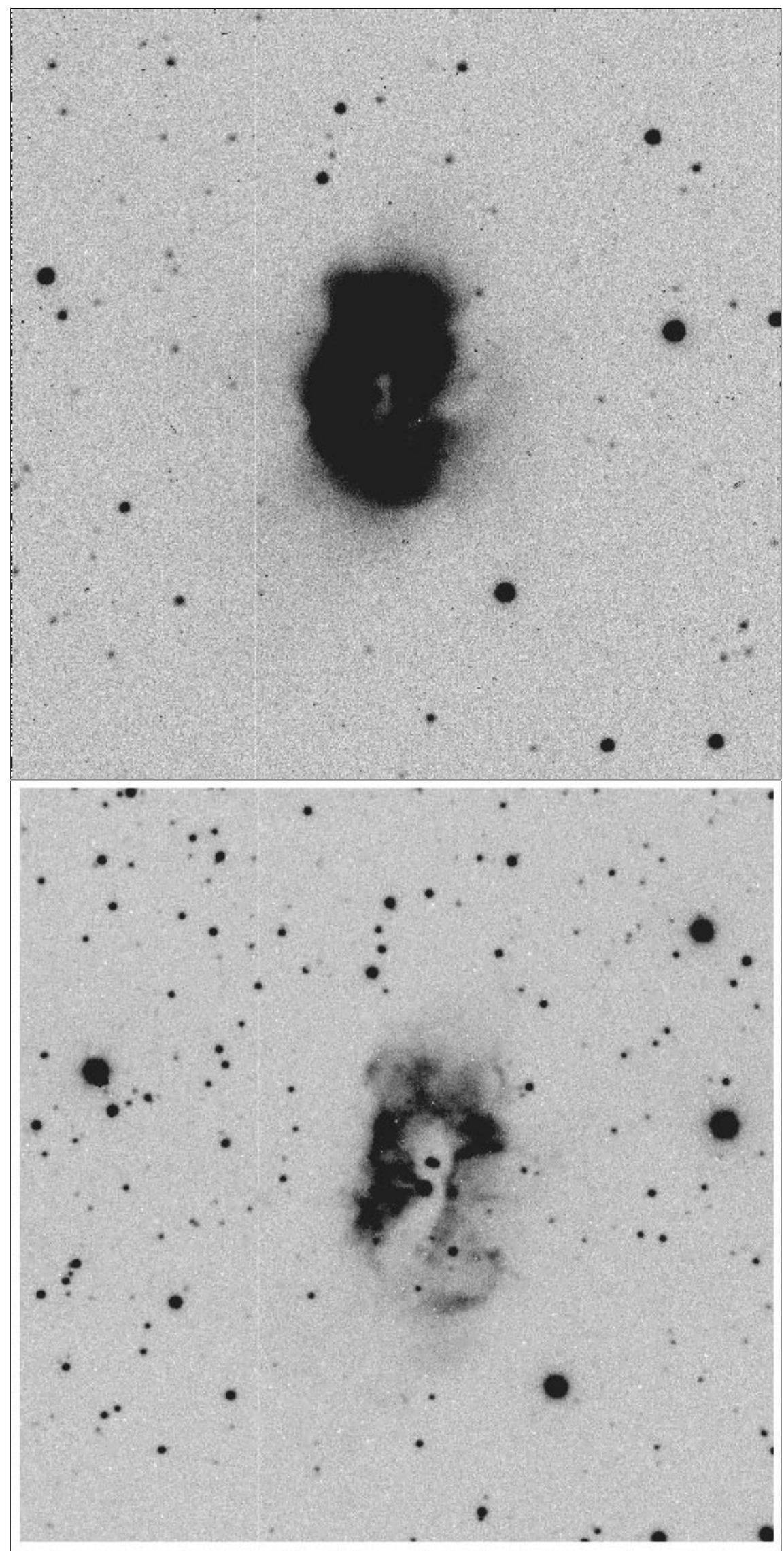

Fig. 3. c) Top: [OIII]5007. The outer halo seen in the two previous emission lines is not detected in [OIII]. d) Bottom: [SII]. A faint emission is detected southwards 


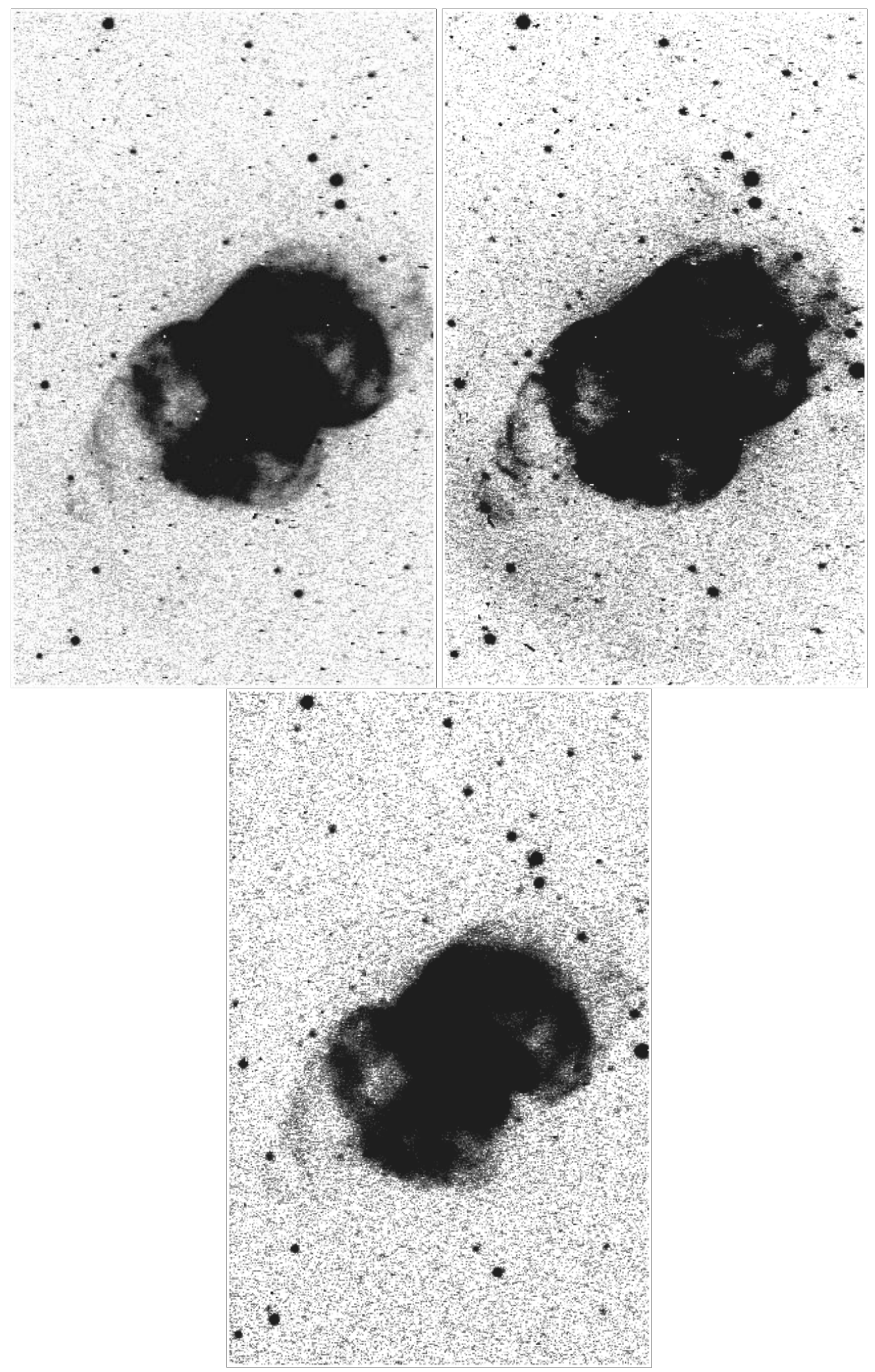

Fig. 4. a) Top left. The limited dimensions of the RCA-CCD detector did not allow to display the eastern region of NGC 650-1. b) Top right: ([NII]). Same remarks as for $\mathrm{H}_{\alpha}$. c) Bottom: The [OIII] image displays the same morphology as in $\mathrm{H}_{\alpha}$ 


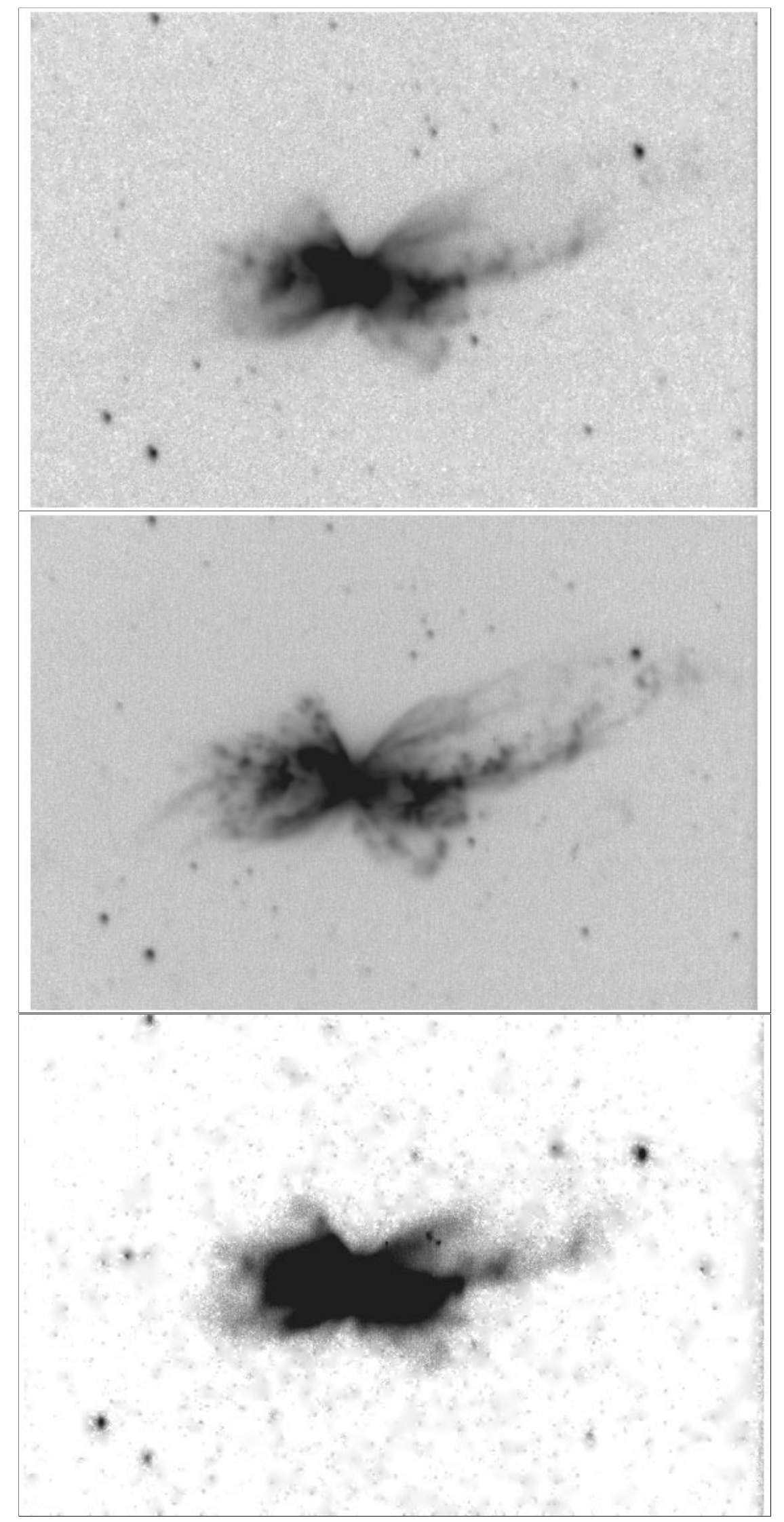

Fig. 5. a) Top: H $\alpha$. NGC 6302 was observed at the MJUO 1-m telescope. North is towards the right and east is at the top. b) Middle: [NII]6583. Same orientation as for $\mathrm{H}_{\alpha}$. The knotty westward emission is more extended in [NII]. c) Bottom: Only the bright core is seen in [OIII] 


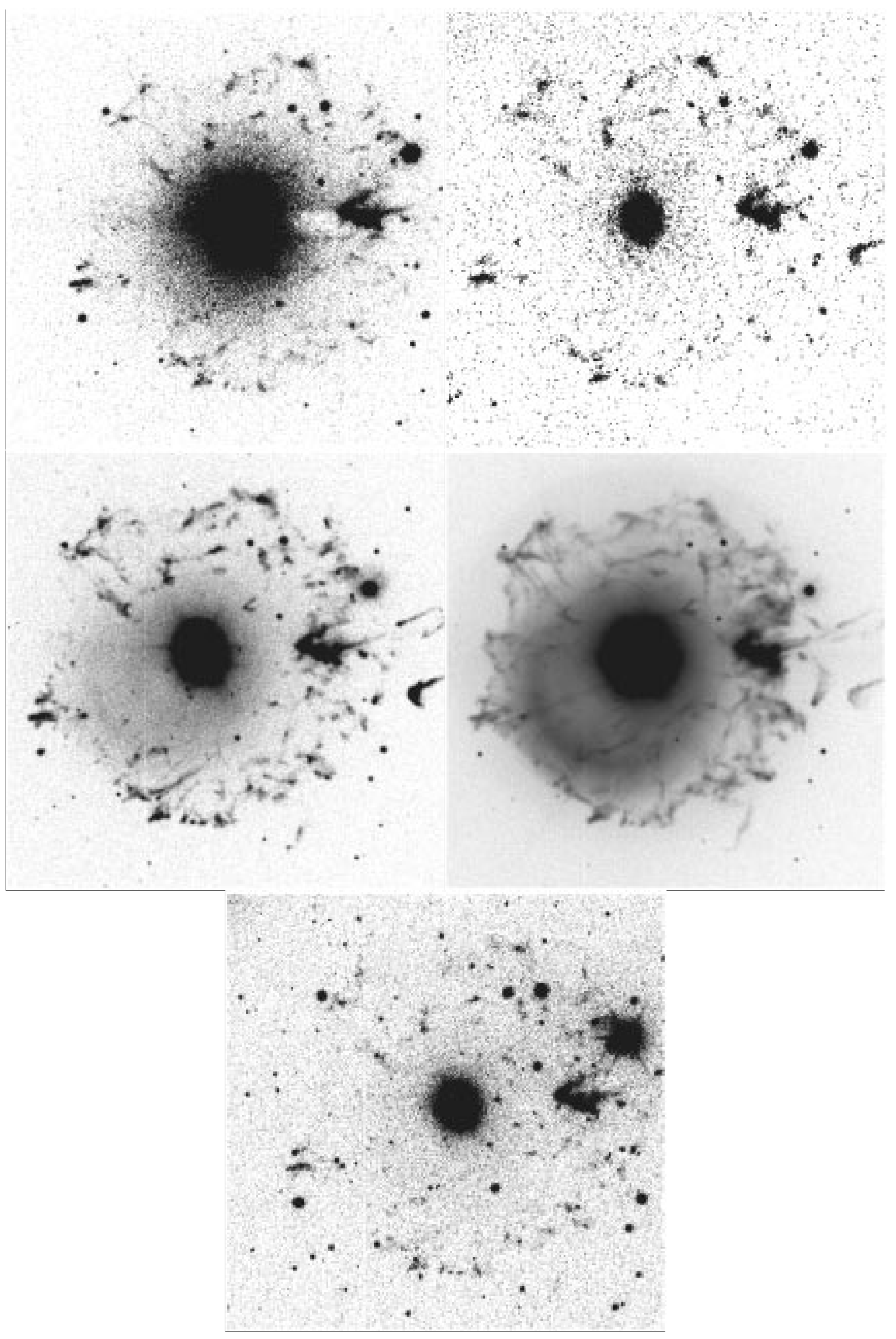

Fig. 6. The low and high ionisation potential ions apparently occupy the same volume. a) Top left: H $\alpha$. b) Middle left: [NII] emission shows a more conspicuous filamentary structure. c) Top right: The [OII] emission region is similar to the [OIII] ones. d) Middle right: $\mathrm{O}^{2+}$ ions are seen in the same region as for [OII] and [SII]. The inner ring in the left side is the ghost image of the bright centre. e) Bottom: The [SII] emission is fairly weak in comparison with the other ionic species 


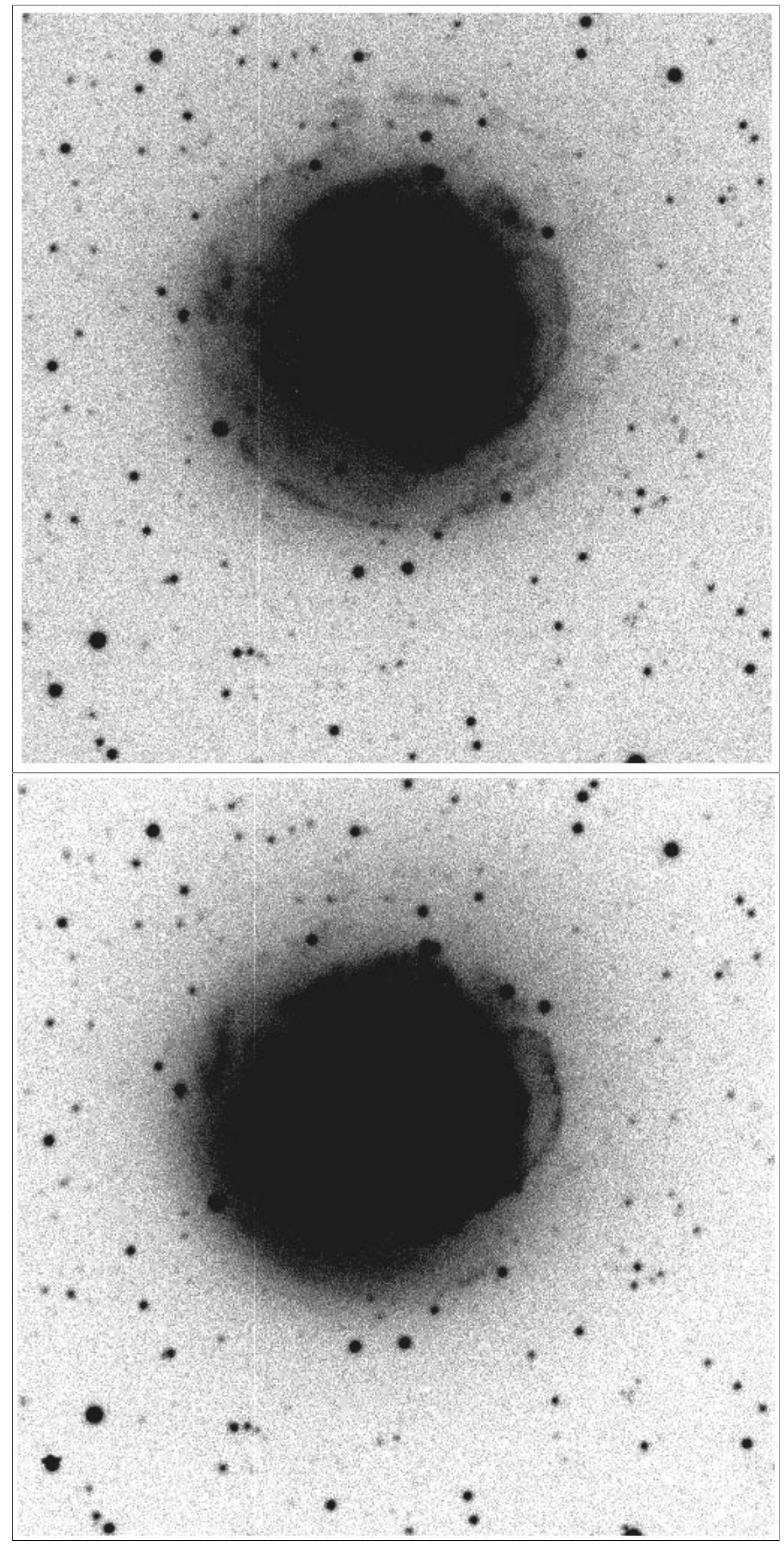

Fig. 7. a) Top: $\mathrm{H} \alpha$ The overall dimensions of the Ring nebula are $260 \times 260$ square arcseconds. The $\mathrm{H}_{\alpha}$ image shows an outer circular "ring" not seen in [NII]. b) Bottom: The outer [NII] emission is more diffuse than in $\mathrm{H}_{\alpha}$ 
spreading over a more extended area $250^{\prime \prime} \times 300^{\prime \prime}-$ $1.2 \times 1.45 \mathrm{pc}$ at the distance of $997 \mathrm{pc}-\left(\mathrm{CKS} 92,1^{\prime \prime}=\right.$ $0.0048 \mathrm{pc}$ ), much larger than quoted so far. The [OIII $] 5007$ image is smaller, limited to the bright $\left(100^{\prime \prime} \times 150^{\prime \prime}\right)$ elliptical ring, without the outer emission detected in the $\mathrm{H}_{\alpha}$ and [NII] images.

\section{4. $N G C 650-1$ : $P N 130-10.1$}

This planetary nebula (Figs. 4) was imaged with the $320 \times 512$ RCA-CCD. The images in $\mathrm{H}_{\alpha},[\mathrm{NII}] 6583 \AA$ and [OIII] $5007 \AA$ display an apparent morphology which shows strong similarities with Sh $1-89$. The overall extension is certainly larger than the dimensions revealed by the CCD in use $\left(\sim 300^{\prime \prime}\right)$.

\section{5. $N G C$ 6302: $P N 349.5+01.0$}

This young bipolar PN is characterized by a high interstellar absorption since the $F$ (radio) $/ F\left(\mathrm{H}_{\beta}\right)$ ratio implies $E(B-V)=0.96$. The excitation is very high (class $10^{+}$); the helium abundance $\mathrm{He} / \mathrm{H}$ is twice $(0.182)$ the average value for known PN. Furthermore, nitrogen $\left(\mathrm{N} / \mathrm{H}=8510^{-5}\right)$ has 10 times the average abundance (CKS92).

Our monochromatic images (Figs. 5) in $\mathrm{H}_{\alpha}, \mathrm{H}_{\beta}$, [NII] $6583 \AA$ and [OIII] $5007 \AA$ obviously reveal a butterfly shape with an "explosive" structure around a bright compact central core separated into two areas by an absorbing lane. The $\mathrm{H}_{\alpha}$ and [OIII]5007 images display similar structures, whereas the [NII]6583 image shows a more knotty morphology. The present observations have detected even larger dimensions $\left(>250^{\prime \prime}\right)$ than that reported by Ashley (1990) (Proc. ASA, 8, 360). The narrowband [NII]6583 filter helps to outline conspicuous filaments more extended (NW) than in $\mathrm{H}_{\alpha}$.

\section{6. $N G C$ 6543: $P N 096.4+29.9$}

This is one of the most outstanding PN among the socalled multiple-shell planetary nebulae, displaying a large faint envelope ( $\sim 5^{\prime}$ in diameter $)$. It is obviously of interest to investigate the morphological nature of the surrounding giant halo. We have observed (Figs. 6) this $\mathrm{PN}$ through the $\mathrm{H}_{\alpha}$, [NII] $6583 \AA$, [OIII] $5007 \AA$, [OII] $3727 \AA$, and $39 \AA$ wide $[\mathrm{SII}]$ filters. All images show conspicuous ejecta far from the main nebula. The global filamentary structure appears up to $270^{\prime \prime}$ from the bright core, in agreement with Middlemass et al. (1989) who considered them as low surface brightness areas and probable density enhancements, illustrating the point that ionizing photons are escaping from the system.

The overall diameter in the observed images is $340^{\prime \prime}$ including the faint outermost structures. This dimension corresponds to $\sim 1.6 \mathrm{pc}$ at the distance given by CKS92
(982 pc). This overall extension raises the question of the age of the nebula. The present estimated value of 3000 years probably involves solely the bright central nebula of $\sim 30^{\prime \prime}$ wide. The actual dimensions of NGC 6543, along with the expansion velocity in the outer region, $10 \mathrm{~km} \mathrm{~s}^{-1}$, would infer a much larger age $\left(\geq 10^{5}\right.$ years). Presumably, we see previous ejecta by the AGB progenitor.

\subsection{The Ring Nebula: NGC 6720 - PN $063.1+13.9$}

The Ring Nebula is certainly one of the most extensively investigated planetary nebula since its discovery (Duncan 1937). Moreno \& Lopez 1987 showed that the Ring Nebula has dimensions definitely larger than the optical $76^{\prime \prime}$ of the bright elliptical core (Moreno \& Lopez 1987; Bryce et al. 1994). Our monochromatic observations (Figs. 7) through narrow $\left(\mathrm{H}_{\alpha},[\mathrm{NII}] 6583\right.$, [OIII] 5007) and moderate $\left(\mathrm{H}_{\beta}, \Delta \lambda=55 \AA\right.$, [SII] $\left.\lambda 6737, \Delta \lambda=39 \AA\right)$ bandwidth filters confirm these outermost features surrounding the filamentary network.

\section{Summary}

We have presented deep monochromatic images of seven type I galactic planetary nebulae. Except for the case of A 39 (where we have not detected outer emission) the observations evidence bipolarity and multiple-shell structures. The linear diameters generally exceed 1 pc. Consequently, the derived ionized mass is often larger than $1 M_{\odot}$. This might contribute to the solution of the mass deficit in planetary nebulae.

Acknowledgements. It is our great pleasure to thank INSU and the G0968 GDR "Milieux Circumstellaires" for travel grant, and our colleagues of the University of Canterbury (Drs. J.B. Hearnshaw, W. Tobin) for their friendly advices. Our thanks also go to Mike Clark for his assistance during the observations at the MJUO telescope. We are indebted to Prof. Michael Dopita of Mt Stromlo for fruitful discussions and corrections to improve the manuscript, to Drs. J. Lequeux, J.M. Deharveng and to the anonymous referee for pertinent comments.

\section{References}

Acker, Ochsenbein F., Stenholm B., Tylenda R., Marcout J., Mischon C., 1993, The Strasbourg-ESO Catalogue of Galactic Planetary Nebulae, ESO Publishing (Acker93)

Balick B., 1987, AJ 94, 671

Bryce M., Balick B., Meaburn J., 1994, MNRAS 266, 721

Cahn J.H., Kaler J.B., 1971, ApJ (CK71)

Cahn J.H., Kaler J.B., Stanghellini L., 1992, A\&AS 94, 399 (CKS92)

Duncan J.D., 1937, ApJ 86, 496

Frank A., Balick B., Icke V., Mellema, G., 1993, ApJ 404, L25 
Jewitt D.C., Danielson B.E., Kupferman P.N., 1986, ApJ 302, 727

Kaler J.B., 1985, ARA\&A 23, 89

Manchado A., Pottasch S.R., 1989, A\&A 222, 219

Middlemass D., Clegg R.E.S., Walsh J.R., 1989, MNRAS 239, 1

Moreno M.A., Lopez J.A., 1987, A\&A 178, 319
Peimbert M., 1978, IAU Symp. 1976, Terzian Y. (ed.). Reidel Publ., p. 215

Peimbert M., 1981, in Physical Processes in Red Giants, Iben Jr. \& Renzini (eds.). Reidel Publ., p. 409

Peimbert M., Serano A., 1980, Rev. Mex. A\&A 5, 9

Peimbert M., Torres-Peimbert S., 1983, IAU Symposium 103, London. Reidel, Dordrecht 